\title{
Respuesta patológica completa en pacientes sometidos a neoadyuvancia en cáncer de recto*
}

\author{
Drs. DANIELLA ESPÍNOLA M. ${ }^{1}$, MARÍA E. MOLINA P. ${ }^{1}$, FELIPE BELLOLIO R. ${ }^{1}$, \\ JOSÉ GELLONA V. ${ }^{1}$, MARIZA BUSTOS C. ${ }^{2}$, ÁLVARO ZÚÑIGA D. ${ }^{1}$ \\ 1 Unidad de Coloproctología, Departamento Cirugía Digestiva, Pontificia Universidad Católica de Chile. \\ 2 Servicio de Radioterapia Centro de Cáncer N.S.E., Facultad de Medicina, Pontificia Universidad Católica de Chile. \\ Santiago, Chile.
}

\begin{abstract}
Pathologic complete response in patients with neoadjuvant chemoradiotherapy for rectal cancer

Background: The standard treatment of locally advanced rectal cancer (RC) of the middle and lower third of the rectum is neoadjuvant chemoradiotherapy (XRQT) follow by oncologic resection. After this treatment in $15-25 \%$ of the cases, the pathologist reports complete pathological response (pCR). Aim: To describe demographic, clinical and survival data of patients with pCR undergoing chemoradiotherapy and radical resection for RC. Material and Methods: Historic cohort study. In a prospectively maintained database between 2000 and 2010, we identified patients with RC, who underwent neoadjuvant chemoradiotherapy according to protocol, followed by radical resection. The preoperative staging was obtained by clinical examination, endoscopy, rectal ultrasound, CT scan of chest, abdomen and pelvis and pelvic MRI. Demographic data, tumor location, time between the end of XRTQ and surgery, postoperative staging (according AJCC) and survival, were collected. Results: 119 patients received preoperative XRTQ, 65\% male, with a mean age of 58 years. The most frequent tumor site was the lower third (63\%). Surgery was performed 8 weeks after the end of XRTQ. Of 119 patients with XRTQ, $15.1 \%$ had a pCR. Overall survival was $75 \%$, and cancerspecific survival was $80.4 \%$ at 5 years in patients without pCR. For patients with pCR, the 5 year survival estimates for overall and cancer specific survival was $100 \%$. We did not identify factors associated with pCR. Conclusions: In this study, pCR was comparable to other larger series reported elsewhere. No factors associated with pCR were identified.
\end{abstract}

Key words: Pathological complete response, rectal cancer, neoadjuvant chemoradiotherapy.

\section{Resumen}

Introducción: El cáncer de recto $(\mathrm{CR})$ de tercio medio e inferior localmente avanzado se trata con radio-quimioterapia (XRTQ) preoperatoria. Luego XRQT y resección quirúrgica, 15-25\% presentan respuesta patológica completa (RPC) de la lesión. Objetivo: Comparar características demográficas, clínicas y sobrevi-

*Recibido el 27 de febrero de 2013 y aceptado para publicación el 22 de marzo de 2013.

Los autores no refieren conflictos de interés

Correspondencia: Dra. Daniella Espínola M. Marcoleta 340, Santiago, Chile. daniellaespinola@gmail.com 
da de pacientes con RPC y respuesta parcial sometidos XRTQ preoperatoria y resección radical. Materiales y Métodos: Estudio cohorte concurrente. En la base de datos de pacientes con CR mantenida prospectivamente, entre 2000-2010, se identificaron pacientes con CR tercio medio e inferior, sometidos XRTQ preoperatoria según protocolo, seguidos de resección radical. Etapificación preoperatoria según: examen clínico, endoscopia, endosonografía rectal, TAC tórax abdomen pelvis y resonancia nuclear magnética de pelvis. Se registraron datos demográficos, localización tumoral, lapso entre término de XRTQ y cirugía, etapificación post operatoria (AJCC), seguimiento y sobrevida. Resultados: 119 pacientes recibieron XRTQ preoperatoria por CR, 65\% hombres. Edad promedio: 58 años. Localización tumoral más frecuente: tercio inferior (63\%). Cirugía se realizó 8 semanas después del término de XRTQ. Etapificación post operatoria: Etapa I 26,1\%, II 34,5\%, III $16,8 \%$, IV 5\% y RPC $15,1 \%$. Sobrevida global $75 \%$, sobrevida específica por cáncer $80,4 \%$ a 5 años. Sobrevida pacientes con RPC fue $100 \%$ a 5 años. No se identificaron factores asociados a RPC. Conclusiones: En este estudio no se logró reconocer factores asociados a RPC. Con las limitaciones que impone el número de pacientes y el seguimiento, se reproducen hallazgos vistos en series más extensas.

Palabras clave: Respuesta patológica completa, cáncer recto, radio-quimioterapia neoadyuvante.

\section{Introducción}

Actualmente, es ampliamente aceptado que el tratamiento del cáncer de recto (CR) localmente avanzado del tercio medio o inferior incluye la radio-quimioterapia (XRTQ), esquema largo: 45 Gy a la pelvis más sobreimpresión de 5,4 Gy en 5 semanas, asociado a 5-fluoruracilo) previo a la cirugía ${ }^{1,2}$. Estudios randomizados han destacado las ventajas y mejores resultados de la XRTQ preoperatoria que ha demostrado tener el potencial de provocar la disminución del tamaño tumoral, inducir cambios favorables en la etapificación de la enfermedad (down staging), aumentar la probabilidad de preservación del esfínter, disminuir la incidencia de recurrencia local, con la posibilidad, además, de lograr respuesta patológica completa (RPC) $)^{3,4}$.

La RPC se define como la imposibilidad de identificar células neoplásicas viables en la pieza operatoria. Un 10-30\% de los pacientes sometidos a XRTQ preoperatoria logran una $\mathrm{RPC}^{5,6 .}$. Hay un subgrupo de pacientes en los que en el estudio de la pieza operatoria se demuestra que no hay tumor en la pared rectal: respuesta mural completa (RMC), pero hay presencia de metástasis ganglionares. Este hallazgo se observa en el 2 a $17 \%$ de aquellos con $\mathrm{RMC}^{7,8}$

Varios estudios sugieren fuertemente que la RPC se relaciona con un mejor control local de la enfermedad, sobrevida libre de enfermedad y sobrevida global $^{5,9,10}$. Desafortunadamente la RPC y RMC son conocidos cuando se obtiene el estudio histopatológico de la pieza operatoria y el único elemento que pudiera anticipar una RPC o una RMC es el examen endoscópico de la lesión, 6 a 8 semanas después de terminada la XRTQ. De manera que es esta respuesta endoscópica completa la que genera la inquietud de contemplar operaciones conservadoras, particularmente cuando la alternativa es la resección abdomino perineal del recto o el riesgo quirúrgico es muy alto. Actualmente, en este escenario es aceptable no intervenir quirúrgicamente estos pacientes esperando que la respuesta clínica también se haya extendido a los ganglios linfáticos ${ }^{11}$.

El objetivo de este estudio es describir las características de los pacientes con RPC en una serie de pacientes sometidos al mismo protocolo de XRTQ preoperatoria y tratar de determinar factores que se asocien a esta condición y objetivar su impacto en la sobrevida y control local de la enfermedad.

\section{Material y Método}

En la base de datos de pacientes con neoplasias colorrectales, mantenida en forma prospectiva, se identificó a todos los pacientes con adenocarcinoma del recto del tercio medio o inferior que fueron sometidos a XRTQ preoperatoria, entre los años 2000 y 2010 en el Hospital Clínico de la Pontificia Universidad Católica de Chile. Se consideró como tercio inferior del recto el segmento entre los márgenes del ano y $7 \mathrm{~cm}$ proximales; tercio medio entre 7 y $11 \mathrm{~cm}$. Se registraron datos demográficos, del tumor, localización, operación practicada y datos del estudio histopatológico de la pieza operatoria. La etapificación preoperatoria se obtuvo por examen clínico, colonoscopia, tomografía axial computada (TC) de tórax, abdomen y pelvis, endosonografía rectal y/o resonancia nuclear magnética $(\mathrm{RMN})$ de pelvis. El protocolo de tratamiento neoadyuvante incluyó la administración de 45 Gy a la pelvis más sobreimpresión de 5,4 Gy en 5 semanas, asociado a $200 \mathrm{mg} / \mathrm{m}^{2}$ de 5 - fluoruracilo en infusión continua o Xeloda $820 \mathrm{mg} / \mathrm{m}^{2}$ dos veces al día durante la radioterapia. La resección quirúrgica se programó para 6 a 8 semanas después de terminada la XRTQ y consistió en la resección quirúrgica radical, esto es, resección total del mesorrecto con o sin preservación esfinteriana (resección anterior o resección abdomino perineal del recto). 
En el estudio anátomo patológico se definió como RPC la ausencia de células tumorales en la pared rectal, mesorecto y ganglios linfáticos de la pieza operatoria. Se denominó respuesta mural completa (RMC) la ausencia de tumor en la pared rectal y ganglios linfáticos con metástasis. La regresión parcial de la lesión se consideró como respuesta parcial (RP).

Se obtuvo un seguimiento completo de los pacientes por medio del registro de los controles regulares con sus médicos tratantes, contactos telefónicos y certificados de defunción en el registro civil.

El análisis estadístico incluyó estadística descriptiva, las variables cuantitativas se expresan en promedio (mínimo, máximo) y se compararon con la prueba $t$ de Student dada su distribución normal. Las variables cualitativas se expresan en números y porcentajes y se compararon mediante $\chi^{2}$ y prueba exacta de Fisher según corresponda. Para el análisis de sobrevida se utilizó el método de Kaplan Meier y test de Log Rank para comparar curvas de sobrevida.

\section{Resultados}

En el período mencionado 119 pacientes recibieron XRTQ preoperatoria por CR de tercio medio o inferior. Cuarenta y dos de ellos mujeres $(35,3 \%)$ y 77 hombres $(64,7 \%)$ con edad promedio de 58 años (29-84).

El estudio histopatológico de la pieza operatoria demostró RP del tumor en 98 pacientes $(82,5 \%), 18$ pacientes $(15,1 \%)$ tuvieron RPC y $3(2,5 \%)$ presentaron RMC.
Las características de los pacientes con respuesta parcial (RP), RPC y de los que presentaron RMC, se muestran en la Tabla 1.

Se puede observar que en 16 de 21 (76\%) pacientes con RMC y RPC tenían tumores localizados en el tercio inferior del recto. Este mismo hecho se observó en el resto de los pacientes de la serie que presentaron algún grado variable de respuesta tumoral (RP).

La etapificación preoperatoria de los pacientes que tuvieron RPC mostró que en su mayoría, 15 de $18(83,3 \%)$ eran tumores T3. Los tres restantes, tumores T2, fueron sometidos a XRTQ preoperatoria considerando la sospecha de ganglios comprometidos según las imágenes, la localización en el tercio inferior y la dificultad de precisar entre tumor T2 y un T3 temprano.

Histológicamente la mayoría de los pacientes de la serie presentaban adenocarcinoma bien o moderadamente diferenciados y sólo 5 (4\%) un tumor poco diferenciado. Entre los pacientes con RMC y RPC 3 $(14,3 \%)$ tenían un tumor poco diferenciado.

Proporcionalmente el número de resecciones anteriores y de resecciones abdominoperineales fue similar entre los pacientes con RPC y RMC y aquellos en los que se observó solamente respuesta parcial. La cirugía se realizó en promedio a las 8 semanas ( $\mathrm{DS} \pm 2,3$ ) posterior al término de la XRTQ en ambos grupos.

La mediana de seguimiento de los pacientes con respuesta parcial fue de 57,6 meses (3,8-135 meses), en el grupo de RMC fue de 57,1 meses (41-126 meses) y en RPC fue de 52 meses (14,8-133,9 meses). El 50\% de los pacientes tienen seguimiento mayor a 5 años. Todos los pacientes con RMC y RPC están

Tabla 1. Respuesta a la radio quimioterapia preoperatoria en 119 pacientes con cáncer de recto

\begin{tabular}{|c|c|c|c|c|}
\hline & $\begin{array}{c}\text { RPC }(n=18) \\
\text { T0 N0 M0 }\end{array}$ & $\begin{array}{c}\text { RMC }(n=3) \\
\text { T0 N1 M0 }\end{array}$ & $\begin{array}{c}\text { RP }(n=98) \\
\text { T1-4 N0-1 M0-1 }\end{array}$ & $\mathbf{P}$ \\
\hline $\operatorname{Edad}^{*}(\bar{x})$ & $57,4(39-80)$ & $59(39-77)$ & $58,8(29-84)$ & 0,911 \\
\hline Sexo (mujeres) & $9 \quad(50 \%)$ & $2(66,7 \%)$ & $31 \quad(31,6 \%)$ & 0,142 \\
\hline $\begin{array}{l}\text { Localización tumor } \\
\text { Medio } \\
\text { Inferior }\end{array}$ & $\begin{aligned} 4 & (22,2 \%) \\
14 & (77,8 \%)\end{aligned}$ & $\begin{array}{l}1(33,3 \%) \\
2(66,7 \%)\end{array}$ & $\begin{array}{ll}39 & (39,8 \%) \\
59 & (60,2 \%)\end{array}$ & 0,314 \\
\hline $\begin{array}{l}\text { T preoperatorio } \\
\text { T2 } \\
\text { T3 } \\
\text { T4 }\end{array}$ & $\begin{aligned} 3 & (44,4 \%) \\
15 & (83,3 \%)\end{aligned}$ & $\begin{array}{l}2(66,7 \%) \\
1(33,3 \%)\end{array}$ & $\begin{array}{rr}5 & (5,2 \%) \\
88 & (90,7 \%) \\
4 & (4,1 \%)\end{array}$ & 0,016 \\
\hline $\begin{array}{l}\text { Operación** } \\
\text { RA } \\
\text { RAP }\end{array}$ & $\begin{aligned} 14 & (77,8 \%) \\
4 & (22,2 \%)\end{aligned}$ & $\begin{array}{l}2(66,7 \%) \\
1(33,3 \%)\end{array}$ & $\begin{array}{ll}80 & (81,6 \%) \\
18 & (18,4 \%)\end{array}$ & 0,563 \\
\hline
\end{tabular}

*Edad expresada en promedio (mínimo y máximo). **RA: resección anterior, RAP: resección abdominoperineal. 


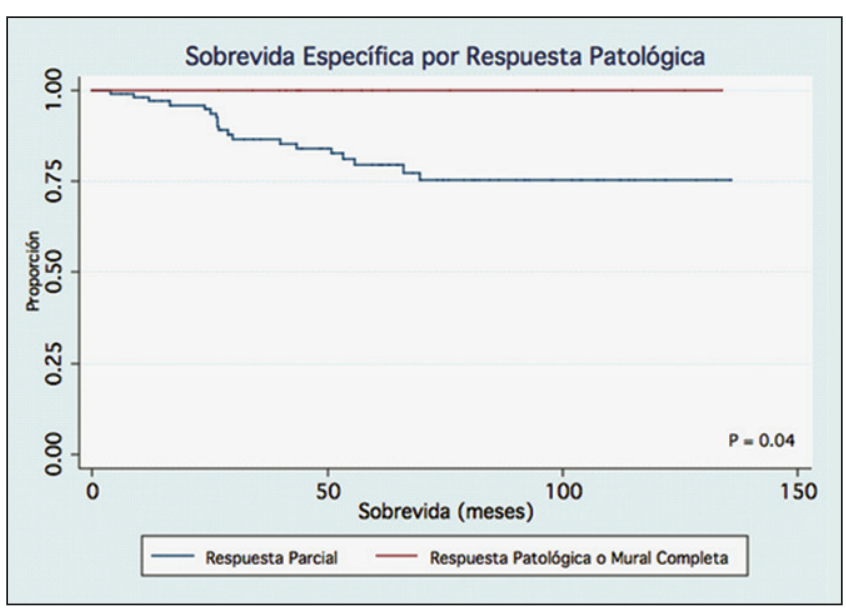

Figura 1. Curva sobrevida.

vivos y sin evidencia de recidiva local o a distancia. En los pacientes con respuesta parcial la sobrevida global a 5 años fue de $85 \%, 82 \%$ y $60 \%$ para los pacientes en etapa I, II y III respectivamente (Figura 1).

En el análisis de los factores de respuesta tumoral (edad, sexo, localización tumoral, $\mathrm{T}$ y $\mathrm{N}$ preoperatorio, histología y grado de diferenciación, tipo de cirugía realizada), no se identificaron factores asociados a RPC.

\section{Discusión}

En este estudio sobre pacientes sometidos a XRTQ preoperatoria por CR avanzado, se observó un $15 \%$ de RPC, lo que es concordante con lo comunicado en otras series, con cifras variables entre $10 \%$ y $30 \%{ }^{5}$. Publicaciones recientes que incluyen un número significativo de pacientes muestran que aquellos con RPC tienen una mejor sobrevida a 5 años y menores tasas de recidiva local. Manteniendo las proporciones, en esta serie, se aprecia el mismo efecto. Se observó que $21(18 \%)$ pacientes no presentan tumor residual en la pared rectal, confirmada por el estudio histopatológico de la pieza operatoria. Sin embargo, 3 de ellos (14\%) tenían metástasis linfáticas en ganglios del mesorrecto (RMC). Este hecho también se ha observado en otros estudios, entre el 2 y $17 \%$ de los pacientes logran una $\mathrm{RMC}^{7,8}$. De manera que la respuesta en la pared intestinal a la radioterapia, no garantiza la ausencia de metástasis linfáticas. De acuerdo a experiencias recientemente publicadas, este subgrupo de pacientes tendría una sobrevida a 5 años menor que la comunicada cuando se comprueba $\mathrm{RPC}^{5}$. Este hallazgo sería una indicación para completar el tratamiento quirúrgico con quimioterapia adyuvante. En nuestra serie, los 3 pacientes en esta situación, no presentan evidencia de recurrencia de tumor con seguimiento variable entre 4 y 10 años.

En este estudio no se logró determinar relaciones entre RPC y el lapso entre el término de la XRTQ y la operación, dado que por protocolo, los pacientes fueron intervenidos entre 6 y 8 semanas sin que hubiera oportunidad de observación en lapsos mayores. En dos grandes series publicadas durante los últimos 5 años se demuestra que el único factor predictivo de RPC es un período de espera mayor a 8 semanas luego del término de la XRTQ ${ }^{12,13}$. En estos estudios, igual que en esta serie, índices como: edad, sexo, localización, etapa del tumor y diferenciación no se asociaron a RPC. Incluso el estudio de algunos marcadores moleculares tampoco ha sido de utilidad ${ }^{13,14}$. En nuestra serie hubo 4 pacientes que fueron sometidos a una resección abdominoperineal cuyo estudio de la pieza operatoria demostró una RPC. Todos ellos sin evidencia actual de recidiva tumoral pero portando una colostomía que compromete su calidad de vida. Desde la perspectiva oncológica no hubo ningún beneficio en practicar una intervención radical por un tumor que regresó en su totalidad. Además habría poco beneficio oncológico en practicar una cirugía radical en un tumor en que la XRTQ lo llevó a un estado patológico T1N0, en cuyo caso la resección local sería la intervención adecuada.

El mensaje que dejan estas observaciones es que la indicación del tratamiento quirúrgico radical basado en la etapificación pre-XRTQ es frágil y puede llevar a intervenciones innecesarias que comprometen la calidad de vida de los pacientes. Esto exige la necesidad de poder determinar la verdadera etapa tumoral después de la XRTQ antes de indicar una cirugía radical y poder así dar lugar a resecciones más localizadas o seguimiento y observación si el control endoscópico después de finalizada la XRTQ no demuestra lesión o sólo una mínima cicatriz.

Las limitaciones de este estudio tienen relación con el reducido número de pacientes con RPC y con su carácter retrospectivo que impidió detectar factores predictores. Entre estos no se analizó el lapso de espera entre el término de la XRTQ y la cirugía y tampoco la extensión circunferencial del tumor, que han sido predictores en algunas series ${ }^{4,12}$.

En conclusión, en esta serie con limitaciones en cuanto al número de pacientes con RPC, se observan los mismos resultados favorables en términos de recidiva 
local y sobrevida que han sido comunicados en series más extensas.

\section{Referencias}

1. Sauer R, Becker H, Hohenberger W, Rödel C, Wittekind C, Fietkau R, et al. Preoperative versus postoperative chemoradiotherapy for rectal cancer. N Engl J Med. 2004;351:1731-40.

2. Fleming F, Pahlman L, Monson J. Neoadyuvant therapy in rectal cancer. Dis Colon Rectum 2011;54:901-12.

3. Janjan NA, Khoo VS, Abbruzzese J, Pazdur R, Dubrow $\mathrm{R}$, Cleary KR, et al. Tumor downstaging and sphincter preservation with preoperative chemoradiation in locally advanced rectal cancer: the MD Anderson Cancer Center experience. Int J Radiat Oncol Biol Phys. 1999;44:1027-38.

4. Kalady M, de Campos-Lobato L, Stocchi L, Geisler D, Dietz D, Lavery I, et al. Predictores factors of pathologic complete response after neoadyuvant chemoradiation for rectal cancer. Ann Surg. 2009;250:582-9.

5. Yeo S, Kim D, Kim T, Chang H, Oh J, Park W, et al. Pathologic complete response of primary tumor following preoperative chemoradiotherapy for locally advanced rectal cancer: long-terms outcomes and prognostic significance of pathological nodal status (KROG 09-01). Ann Surg. 2010;252:998-1004.

6. Smith FM, Waldron D, Winter DC. Rectum-conserving surgery in the era of chemoradiotherapy. Br J Surg. 2010;97:1752-64.

7. Bujko K, Nowacki MP, Nasierowska-Guttmejer A, Kepka L, Winkler-Spytkowska B, Suwinski R, et al. Polish Colorectal Stydy Group. Prediction of mesorectal nodal metastases after chemoradiation for rectal cancer: results of a randomized trial: implication for subsequent local excision. Radiother Oncol. 2005;76:234-40.

8. García-Aguilar J, Hernández de Anda E, Sirivongs P, Lee SH, Madoff RD, Rothenberg DA. A pathologic complete response to preoperative chemoradiation is associated with lower local recurrence and improved survival in rectal cancer patients treated by mesorectal excision. Dis Colon Rectum 2003;46:298-304.

9. Guilliem JG, Chessin DB, Cohen AM, Shia J, Mazumdar M, Enker W, et al. Long-term oncologic outcome following preoperative combined modality therapy and total mesorectal excision of locally advanced rectal cancer. Ann Surg. 2005;241:829-36.

10. Bonnen M, Crane C, Vauthey J, Skibber J, Delclos ME, Rodriguez-Bigas $\mathrm{M}$, et al. Long-term results using local excision after preoperative chemoradiation among select T3 rectal cancer patients. Int Radiat Oncol Biol Phys. 2004;60:1098-105.

11. Habr-Gamma A, Pérez RO, Sao Juliao GP, Proscurshim I, Gamma-Rodrigues J. Nonoperative approaches to rectal cancer: a critical evaluation. Semin Radiat Oncol. 2011;21:234-9.

12. Das P, Skibber JM, Rodríguez-Bigas MA, Feig BW, Chang GJ, Wolff RA, et al. Predictors of tumor response and downstaging in patients who receive preoperative chemoradiation for rectal cancer. Cancer 2007;109:1750-5.

13. Rödel C, Grabenbauer GC, Papadopoulos T, Bigalke M, Günter K, Schick C, et al. Apoptosis as a cellular predictor for histopathologic response to neoadyuvant chemoradiotherapy in patients with rectal cancer. Int $\mathrm{J}$ Radiat Oncol Biol Phys. 2002;52:294-303.

14. Kuremsky JG, Tepper JE, McLeod HL. Biomarkers for response to neoadyuvant chemoradiotherapy for rectal cancer. Int J Radiat Oncol Biol Phys. 2009; 74:673-88.

15. Huges R, Glynne-Jones R, Grainger J, Richman P, Makris A, Harrison M, et al. Can pathological complete response in the primary tumour following pre-operative pelvic chemoradiotherapy for T3-T4 rectal cancer predict for sterilization of pelvic lymph nodes, a low risk of local recurrence and the appropriateness of local excision? Int J Colorectal Dis. 2006;21:11-7. 